of conformations that maintain the atoms in the ester grouping coplanar (Fig. 1). The large number of conformations can be appreciated by recalling that there is essentially free rotation about the $\mathrm{O}-\mathrm{C}$ bond, that rotation about the bond between the $\alpha$ and $\beta$ carbons also occurs readily ${ }^{3}$ and that hydrogen bonds involving oxygen as the acceptor have few, if any, geometric restrictions ${ }^{4}$.

WILLIAM H. BEERS EDWARD REICH

The Rockefeller University,

New York,

New York 10021

Received April 13, 1971.

1 Chothia, C., and Pauling, P., Nature, 229, 281 (1971).

2 Beers, W. H., and Reich, E., Nature, 228, 917 (1970).

3 Burgen, A. S. V., Ann. Rev. Pharmacol., 10, 7 (1970).

${ }^{4}$ Pauling, L., The Nature of the Chemical Bond, third ed. (Cornell University Press, Ithaca, 1960).

\section{Presence and Function of Disclinations in Surface Coats of Unicellular Organisms}

IF a cell which is topologically a sphere is covered with a coat composed of identical units arranged to form a surface crystal, this crystal necessarily ${ }^{1}$ contains screw disclinations of total strength +2 complete rotations. Harris and Scriven ${ }^{2}$ draw attention to the micrograph of a marine bacterium, Nitrosomonas, published by Watson and Remsen ${ }^{3}$, on the cover of Science, showing a coat of apparently spherical units in close packing. They say that intrinsic disclinations are strongly indicated. We have undertaken further analysis of the disclination structure of this coat.

The structure clearly contains a grain boundary which terminates in the visible part of the coat. Such a termination is a partial disclination. We can show that the visible part of the coat, including the two regions which have apparently been removed in preparation, contains just two disclinations each of strength $+1 / 6$. Fig. 1 reproduces the micrograph of Watson and Remsen, with the addition of lines indicating the continuous variation of a lattice direction in the crystal. To analyse the disclination structure, a point was taken that lay between the termination of the grain boundary and the margin of the adjacent missing region. The lattice direction at this point which lies roughly parallel to the long axis of the photograph was taken as the reference direction; this was also assumed to be the long axis of the organism. From this point, two circuits were described, each encircling one of the missing regions counter-clockwise. The lattice direction followed continuously round the circuit has rotated by $\pi / 3$ counter-clockwise when the circuit closes. Each circuit therefore contains screw disclinations of total strength $+1 / 6$. One disclination is dissociated, and one of its partial disclinations appears as the termination of a grain boundary; the other disclination lies completely within the region which has been removed in preparation.

A disclination of strength $(6-n) / 6$ has $n$-fold rotational symmetry. Thus the addition of two such disclinations, one at each pole, would account for the necessary total strength $+12 / 6$ and would fit an assumed $n$-fold symmetry of the cell. There are exactly two values for $n$ that imply disclinations all of the same strength: $n=5$, with a disclination of strength $+1 / 6$ at each pole, and $n=6$ with no disclinations at the poles. The case $n=7$ requires fourteen disclinations of strength $+1 / 6$ and two of strength $-1 / 6$; all other possible values of $n$ require disclinations at the poles with strengths of magnitude greater than the minimum. Dislocation glide provides a

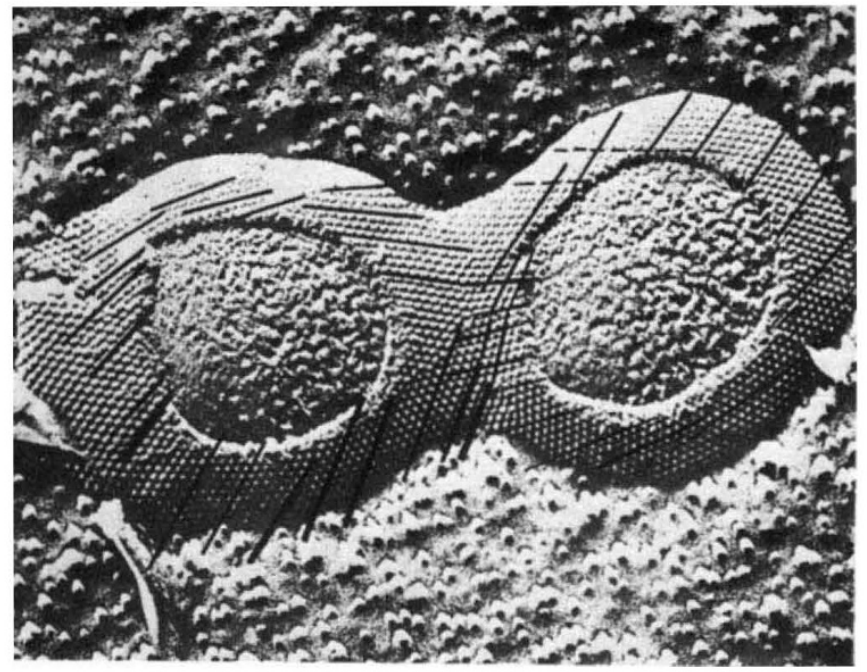

Fig. 1 Micrograph of the coat of Nitrosomonas with additional lines indicating the continuous variation in the lattice direction in the crystal.

mechanism whereby disclinations can dissociate into disclinations of lower strength and move to lower the total strain energy $^{2}$. Therefore one expects all values of $n$, other than 5 and 6 , to be unlikely. In the micrograph there is no evidence for flattening at the poles as is required if $n=6$. Thus one is left with the most probable result that $n=5$, that is, that there are twelve disclinations each of strength $+1 / 6$, including one at each pole.

While the presence of disclinations in coats of this kind is a geometrical necessity, they have no obvious direct biological function. They can, however, act as sources of edge dislocations $^{2}$, and edge dislocations are the natural growth points in a surface crystal ${ }^{4}$. This suggests the intriguing possibility of the chemotherapy of diseases associated with the growth of cells having membranes of this kind by the mechanism known to metallurgists as Cottrell locking. A suitable small molecule will be incorporated into the structure at the core of an edge dislocation in preference to an additional unit of the membrane. Further growth at this point is stopped.

F. R. N. NABARRo

Physique des Solides,

Faculté des Sciences,

Paris XI-Orsay

W. F. HARRIS

Department of Chemical Engineering,

University of the Witwatersrand,

Johannesburg

Received April 26, 1971.

1 Harris, W. F., in Fundamental Aspects of Dislocation Theory (edit. by Simmons, J. A., et al.) (National Bureau of Standards, Washington, DC, in the press).

${ }^{2}$ Harris, W. F., and Scriven, L. E., J. Appl. Phys. (in the press).

3 Watson, S. W., and Remsen, C. C., Science, 163, 685 (1969).

${ }^{4}$ Harris, W. F., and Scriven, L. E., Nature, 228, 827 (1970).

\section{Daucic Acid and its Occurrence in Plants}

DURING anion-exchange chromatography of extracts of biological materials, acidic non-nucleotide contaminants of the nucleotide fraction have often been encountered. They are usually identified by their characteristic ultraviolet absorption maxima. Thus the leaves of maize (Zea mays) were shown to 